\title{
A kind of packet reordering analysis model in concurrent multipath transmission
}

\author{
Yang Tao ${ }^{1, a}$, Jiayong Zhang ${ }^{2, b}$, Xuanrui Xiong ${ }^{3, c}$ \\ ${ }^{1,2,3}$ School of Communication and Information Engineering, Chongqing University of Posts and \\ Telecommunications, Chongqing, 400065, China \\ ataoaoyang@cqupt.edu.cn, bjiayongzhang987@foxmail.com, ’xiongxr@cqupt.edu.cn
}

Keywords: concurrent multipath transmission, packet reordering, cumulative probability distribution function

\begin{abstract}
Focus on the problem of packet reordering in concurrent multipath transmission which lead to an unnecessary fast retransmit and increase in network load, reduces the utilization of network resources. This paper put forward a kind of packet reordering analysis model in concurrent multipath transmission based on the cumulative probability distribution function, besides, a constraint conditions are derived for data packets to be transmitted in order. And the calculation formula of random order probability of data packet in concurrent multipath transmission.
\end{abstract}

\section{Introduction}

In the scene of the concurrent multipath transmission, even in a ideal situation, it is assumed that the upper application with rapid processing capacity of data packet arriving at the receiving end, the phenomenon of packet reordering will also unavoidable. The research in this field now is mainly to make a modeing analysis of the transmission performance of concurrent multipath transmission system in the packet reordering. And this will provide theoretical reference of improving the performance of concurrent multipath transmission system to further reduce the disorder phenomenon.This paper make a deep research of packet reordering problem in concurrent multipath transmission system, put forward a more reasonable packet reordering analysis model. Compare to the existing research, this model take the queue delay and transmission delay into account in order to increase the accuracy and validity of the model.

\section{Research on packet reordering problem in concurrent multipath transmission system}

\subsection{Definition of packet reordering Section Headings.}

The RFC4737[1] had given a definition of packet reordering, and developed a variety of the evaluation criterias to quantify the severity of the disorder.

Packet reordering is defined as follows:

$S$ : Transmission serial number of the sending end assigned to the data packet;

$I$ : The serial number of the data packet that the receiving end receives;

$s[i]$ :The No.i group transmission serial number that the receiving end receives;

$j$ : The smallest integer satisfying the condition of $s[j]>s[i]$ in the top i group that the receiving end receives, besides, $j<i$;

If $s=i$, then the No.i packet is received at a sequence, and packet reordering is not occurred;

Otherwise, if $s \neq i$, then packet reordering is occurred when the receiving end received the No. $\mathrm{i}$ packet, we use " $e$ " to express the reordering degree of group $s[i]$.

\subsection{Reason of packet reordering}

In heterogeneous network environment, the path used in concurrent multipath transmission may belong to different access networks, the different network characteristics lead to transmission delay and bandwidth differences in different paths, The packets on the path of the smaller delay will reach the receiving end earlier than the other paths. The packets arrives early could not be processed in order to the upper application, This part of the packet will be stored in the buffer zone of the receiving 
end, It will submit to the upper application in order when the packets arrive which transmission serial number is small. This is the reason of packet reordering.

\section{Modeling and analysis of packet reordering}

\subsection{Model Description}

Paper [2] points out that the IP data packet is a basic property of the network link path, the problem of packet reordering occurs in the process of transmission in the parallel multi path, mainly caused by the delay difference between multiple concurrent link. In this paper, we draw on the experience of the end-to-end packet reordering performance analysis model in paper[3], and the concurrent multipath transmission system is abstracted as the model shown in Fig.2.

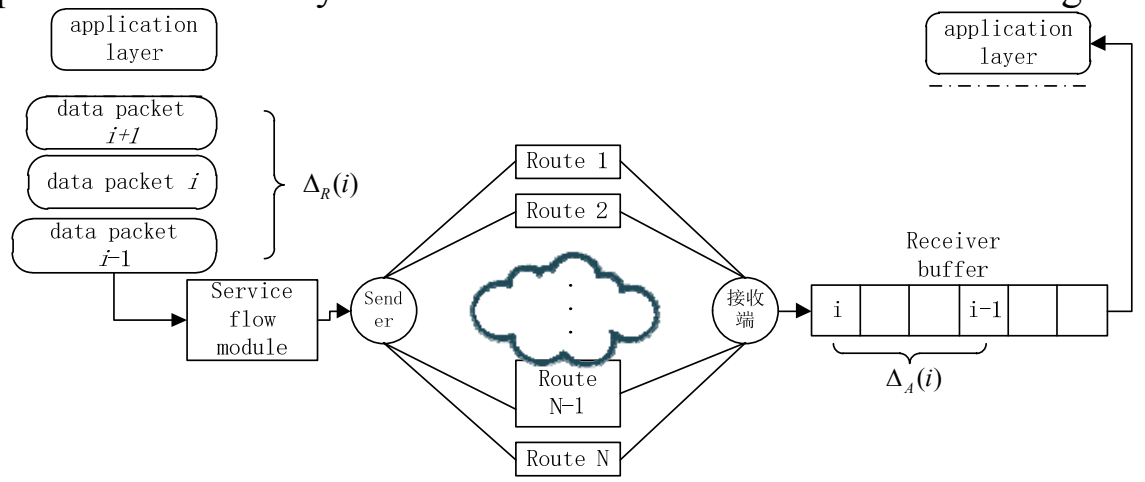

Fig. 2 analysis model of the performance of multi path parallel transmission

\section{Sending End and Receiving End}

As shown in Fig.2, there are $\mathrm{N}(N \geq 2)$ independent transmission path between the sending end and the receiving end, and they are marked as $L_{1}, L_{2}, \ldots, L_{k}, \ldots L_{N}$, besides, the end-to-end transmission delay of each path satisfied the condition of $d_{1} \leq d_{2} \leq \ldots d_{k} \ldots \leq d_{N}$. The traffic flow from the application layer is divided into different sizes of data packets after arrival of the sending end of the business shunt module, each packet is independent of each other in the process of transmission, And it will select one of the $\mathrm{N}$ path in a certain way to send according to a certain data scheduling algorithm. We mark the probability that a data packet select $L_{k}$ as the transmission link path is $p_{k}$, That is to say, the data packet will be distributed to the link path whose end-to-end delay is $p_{k}$ as a $p_{k}$ probability to transmit. Data split scheduling strategy is described as:

$$
P=\left\{p_{1}, p_{2}, \ldots, p_{N} \mid \sum_{i=1} p_{i}=1\right\}
$$

Among them, $p_{i}$ represents the probability of selecting the path $L_{i}$ as the packet delivery path.

2. End-To-End Transmission Delay

The packet reordering phenomenon occurred in receiving end, that is to say, the data packets out of order whose number is continuous must come from different transmission paths. For the convenience of further theoretical analysis, the variables that need to be used in the analysis are as follows:

$R(i)$ - The moment that data packet $i$ arrived at the sending end.

$\Delta_{R}(i)$ - - The time interval between packet $i-1$ and packet $i$ when they arrived at the sending end.

$S(i)$ - - The sending delay that data packet i experienced.

$T(i)$ - The moment that packet i leave the sending end, $T(i)=R(i)+S(i)$.

$\Delta_{T}(i)$ - The time interval between packet $i-1$ and packet $I$ when they left the sending end.

$d(i)$ - The transmission delay of packet I in the process of network transmission.

$A(i)$ - - The moment that packet I arrive at the receiving end, $A(i)=T(i)+d(i)$.

$\Delta_{A}(i)$ - - The time interval between packet $i-1$ and packet $i$ when they arrived at the receiving end. 


\subsection{Theoretical Analysis}

In order to simplify the process of theoretical derivation, the following analysis process temporarily ignore the effects of $S(i)$, data packets are ordered from the development of sending, transmission time and transmission sequence number of the packet transmission sequence number is proportional to the larger data packets when it is late. Therefore, to ensure that the data packet in order to arrive at the receiving end, just let the two adjacent data packets at the receiving end of the meeting:

$$
\forall i \geq 0, \Delta_{A}(i)=A(i)-A(i-1) \geq 0
$$

And then,

$$
\begin{aligned}
& T(i)+d(i) \geq T(i-1)+d(i-1) \\
& d(i) \geq d(i-1)-(T(i)-T(i-1))=d(i-1)-\Delta_{T}(i)
\end{aligned}
$$

Therefore,

We can obtained from the deduction above: when $d(i) \geq d(i-1)-\Delta_{T}(i)$, then they can arrive at the receiving end in order. It can be seen from the formula (3) that the occurrence of the packet disorder is related to the path propagation delay and the transmission interval.

Using the formula (3) to analyze the probability of the occurrence of random sequence in the multi path parallel transmission. Divided into the following two kinds of situations to discuss:

1. $\Delta_{T}(i) \geq d_{N}-d_{1}$

At this point, $\Delta_{T}(i)$ is greater than the maximum propagation delay difference in all paths.so, that will be $d(i-1)-\Delta_{T}(i) \leq d(i-1)-\left(d_{N}-d_{1}\right) \leq d_{1} \leq d(i)$, Whether the data packet $i$ select which one of the transmission link to carry on, all of the data packet will be able to arrive at the order, the probability of occurrence disorder in the process of the data packet transmission is $p\left(\Delta_{T}(i)\right)=0$.

2. $\Delta_{T}(i)<d_{N}-d_{1}$

In a longer period of time, in the process of sending a large number of data packets, the link selectiong of the adjacent two packets $i-1$ and $i$ must satisfied the independent and identically distributed. Assume that the packet $i-1$ is assigned to the link $L_{l}$ for transmission, that will be $d(i-1)=d_{l}$, besides, $d(i-1)-\Delta_{T}(i)=d_{l}-\Delta_{T}(i)<d_{l}$, we use link $L_{l}, L_{l+1}, \ldots, L_{N}$ to transmit data packets $i$ and this could avoid the occurrence of the phenomenon of data disorder. Formula as shown in (5):

$$
p=p_{1} \cdot 0+\ldots+p_{k} \cdot\left(\sum_{j=1}^{k-1} p_{j}\right)+\ldots+p_{N} \cdot\left(1-p_{N}\right)=\sum_{i=1}^{N}\left(p_{i} \cdot \sum_{j=1}^{i-1} p_{j}\right)
$$

In order to describe the distribution characteristics of the end to end transmission delay of data packets in the process of multipath parallel transmission, the cumulative probability distribution function of the transmission delay of each packet is defined as $D(d)$ :

$$
D(d)=\sum_{d_{k}<d} p_{k}=\left\{\begin{array}{lc}
0 & d \leq d_{1} \\
p_{1} & d_{1} \leq d \leq d_{2} \\
p_{1}+p_{2} & d_{2} \leq d \leq d_{3} \\
\cdots & \ldots \\
1 & d>d_{N}
\end{array}\right.
$$

The physical meaning of $D(d)$ is the probability was chosen that the selected transmission path end to end transmission delay is less than $d$.

Therefore, in the premise of the path transmission data packet $i-1$ which has been determined to select the transmission delay for $d(i-1)$, the probability of the constraint condition that the transmission path chosen by the packet $i$ can satisfy the formula (4):

$$
P\left(\Delta_{T}(i)\right)=\sum_{k=1}^{N} p_{k} \cdot D\left(d(i-1)-\Delta_{T}(i)\right)
$$


From the analysis above, we can know that the situaiton we discussed in 1 is a special case of 2, so the multi path parallel transmission system in the probability of data packet disorder can be unified using the formula (7) to calculate.

Next, on the basis of the formula (7), the increase of the transmission delay is considered. So the data packet in the formula (7) is carried out by the time interval of the sending end:

$$
\Delta_{T}(i)=T(i)-T(i-1)=(R(i)+S(i))-(R(i-1)+S(i-1))=\Delta_{R}(i)+S(i)-S(i-1)
$$

Then, we derive the transmission delay of $i$ and $i-1$, respectively, on the basis of the results of the transmission path selection of the known packet $i-1$.

Transmission delay is defined in this paper by the sending end of the queue waiting delay and transmission delay is composed of two parts, in a network data packet queuing problem, most of the existing research using queuing theory model analysis ${ }^{[5]}{ }^{[6]}$. The queuing theory analysis of transmission delay.

Assuming that the arrival process of sending data packet satisfies Poisson distribution, the packet arrival rate is $\lambda$, the size of the data packet is subject to the negative exponential distribution, the capacity of the data packet is subject to the negative exponential distribution, and the transmission rate on the $k$ parallel path is $\mu_{k}$, and the capacity of the transmitter is limited. The data distribution module in sending end distribute arriving packets to the sending queue of $\mathrm{N}$ paths for concurrent transmission, the data distribution on the path of path $k$ is assigned as $\lambda_{k}$, there will be $\lambda_{k}=p_{k} \cdot \lambda$ and $\lambda_{k}=p_{k} \cdot \lambda$. The arrival process of the data packets on the $\mathrm{N}$ link is also satisfied with the Poisson distribution. Therefore, packets in each concurrent chains on the queuing process can be seen as M / M / 1 queueing system with a finite capacity queuing problem. Fig. 2 multipath parallel transmission scenarios can be further simplified to Fig. 3 shows the queueing theory model.

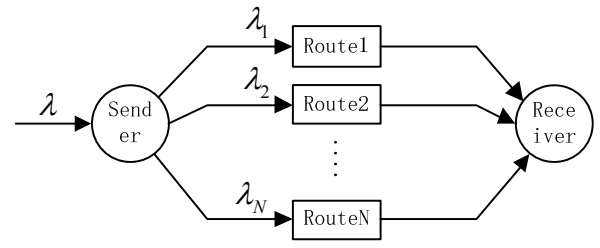

Fig. 3 Packet distribution model in sending

The average length of stay in the queuing system is: $\quad \bar{t}=\frac{1}{\mu-\lambda}$

Therefore, the transmission delay experienced by the data packet $i$ can be estimated by the average length of stay in the $\mathrm{M} / \mathrm{M} / 1$ queuing system, marked as:

$$
S(i)=\sum_{k=1}^{N} p_{k} \frac{1}{\mu_{k}-\lambda_{k}}
$$

The transmission path selection results of data packet $i-1$ is known, marked as:

$$
S(i-1)=\frac{Q(i-1)+M T U}{B(i-1)}
$$

Among them, $Q(i-1)$ represents the queue length on the transmission path of the packet $i-1$, $B(i-1)$ represents the bandwidth of the data packet $i-1$, and $M T U$ is the length of a maximum transmission unit.

Therefore, the formula (8) can be further expressed as:

$$
\Delta_{T}(i)=\Delta_{R}(i)+S(i)-S(i-1)=\Delta_{R}(i)+\sum_{k=1}^{n} p_{k} \frac{1}{\mu_{k}-\lambda_{k}}-\frac{Q(i-1)+M T U}{B(i-1)}
$$


Combination formula (7) and formula (12), considering increasing the impact of the transmission delay, the probability of the occurrence of random sequence in the transmission process of the data packet $i$ can be further expressed as the premise of the data packet $i-1$ transmission:

$$
\left\{\begin{array}{l}
P\left(\Delta_{T}(i)\right)=\sum_{k=1}^{n} P_{k} \cdot D\left(d(i-1)-\Delta_{T}(i)\right) \\
\Delta_{T}(i)=\Delta_{R}(i)+\sum_{k=1}^{n} p_{k} \frac{1}{\mu_{k}-\lambda_{k}}-\frac{Q(i-1)+M T U}{B(i-1)}
\end{array}\right.
$$

We can learn from the formula (13) and its analysis process above, the strategy of path selection and the time interval between the data packets and the data packets are greatly influenced by the data disorder. When the satisfying formula (4) is satisfied, the multipath parallel transmission system under the ideal condition can not be affected by the disorder. Therefore, in order to reduce the data disorder phenomenon, we must increase the waiting time before the data packets were sent to $\operatorname{control} \Delta_{T}(i)$ to satisfied the conditions of the data packet transmission in order, to avoid the disorder by the way of sacrificing the transmission efficiency.

\section{Simulation analysis of data disorder model}

\subsection{Introduction to simulation environment and parameters}

In this paper, the simulation results are verified by using NS-2.35, and the communication protocol of simulation experiment is adopted to support the SCTP-CMT protocol of multi path parallel transmission.

Detailed parameter settings are shown in Table 1:

Table.1 Parameters of simulation experiment

\subsection{Simulation result analysis}

\begin{tabular}{c|c}
\multicolumn{2}{c}{ Table.1 Parameters of simulation experiment } \\
\hline simulation software & NS-2.35 \\
\hline transport protocol & SCTP-CMT \\
\hline Appl ication types & FTP \\
\hline bandwidth & $0.1 \mathrm{Mbps}$ \\
\hline End to end de lay & $\left(10+10^{*}\right.$ Link number)ms \\
\hline Rece ive buffer & 64KB \\
\hline Maximum data load & 1468 bytes \\
\hline MTU & 1500 bytes \\
\hline Path number & scene 1:6, scene 2: 8, scene 3: 10 \\
\hline Retransmission \\
strategy
\end{tabular}

This paper simulated with NS-2.35 simulation software, respectively in 6,8 and 10 link concurrent transmission scenarios of multi-path parallel transmission of data packet reordering probability simulation, every scene simulation experiments are running 50 times, fetch packet chaotic sequence probability average value as the final result.

Fig. 5 shows the comparison results of three parallel transmission scenarios with different number of links. Figure in blue hollow dot curve said without considering transmission delay before Gao Chong reordering models derived from the analysis of numerical results, the solid red dot curve said consider increasing the transmission delay after the numerical results, the black line is obtained through NS-2 simulation experiments data packet average disorder rate curve. In this paper, the data packet arrival time interval of the simulation diagram is from $10 \mathrm{~ms}$, which increases in turn, and the probability curve of the data packet is reduced to 0 .

It can be seen from the comparison of the simulation and the model that the probability of random order increases when the number of links increases and the distance of the arrival of the link is equal. 
This is because according to the experiment of path delay setting, increased as the number of concurrent path, the maximum delay on all paths difference will be more and more, so the data packet transmission process occur chaotic sequence probability is increased. At the same time, with the increase of the number of concurrent paths, the solution of the model is more and more close to the actual simulation results, which shows that the proposed model can effectively estimate the probability of random sequence in the multi path concurrent transmission system. Furthermore, when the addition of sending delay effect are taken into account, the curves of model solution was more close to the actual simulation results curve than before, witch indicating that the data disorder order model increase considering the effects of transmission delay improves the accuracy of the original model.

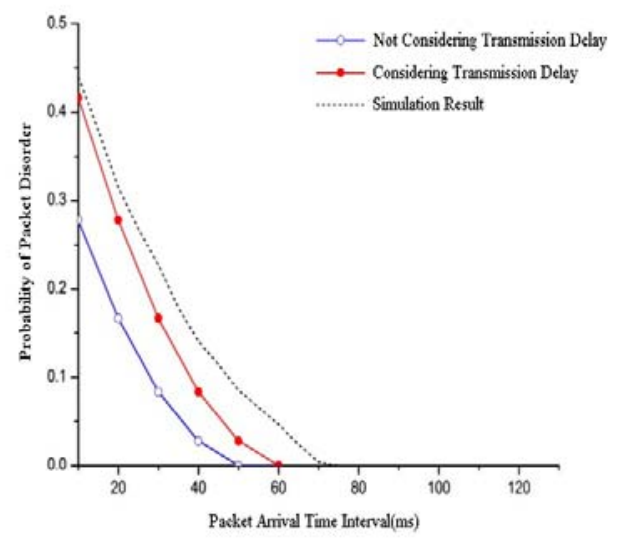

(a)Six link concurrent transmission scenarios scenarios

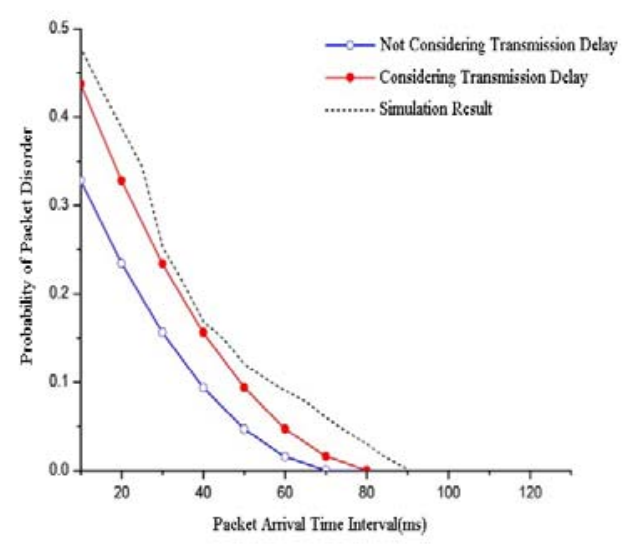

(b) Eight link concurrent transmission

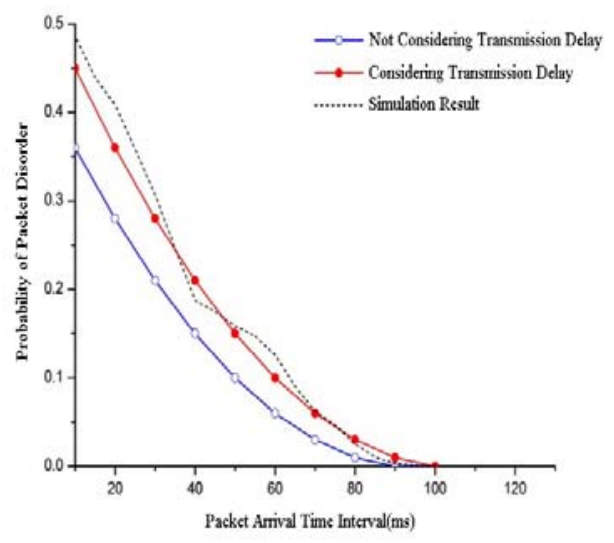

(c) Ten link concurrent transmission scenarios

Fig. 5 simulation results of random sequence of packets in different simulation scenarios

\section{5 conclusion}

Packet reordering is the main factors to suppress the improvement of the performance of multipath parallel transmission system . This paper proposes the multi-path parallel transmission system disorder analysis model based on transmission delay cumulative probability distribution function, which improving the consideration of transmission delay effect to further improve the accuracy of the model on the basis of existing research. Finally, simulation results verify the validity of the model, and provide the necessary theoretical support for the study of the subsequent data disorder. 


\section{Reference}

[1]. Morton A, Ciavattone L, Ramachandran G, et al. RFC4737, Packet reordering metrics[S]. IETF, 2006.

[2]. Leung K C, Lai C, Li V O K, et al. A packet-reordering solution to wireless losses in transmission control protocol[J]. Wireless networks, 2013, 19(7): 1577-1593.

[3]. Gao Chong, Ling Zhihao, Yuan Yingfeng. Packet reordering analysis for concurrent multipath transfer[J]. International Journal of Communication Systems, 2014, 27(12): 4510-4526.

[4]. Parekh A K, Gallager R G. A generalized processor sharing approach to flow control in integrated services networks: the single-node case[J]. IEEE/ACM Transactions on Networking (ToN), 1993, 1(3): 344-357.

[5]. Bobarshad H, van der Schaar M, Aghvami A H, et al. Analytical modeling for delay-sensitive video over WLAN[J]. IEEE Transactions on Multimedia, 2012, 14(2): 401-414.

[6]. Gamini Abhaya V, Tari Z, Zeephongsekul P, et al. Performance Analysis of EDF Scheduling in a Multi-Priority Preemptive M/G/1 Queue[J]. Parallel and Distributed Systems, IEEE Transactions on, 2014, 25(8): 2149-2158.

[7]. 周昫槃. 通信网理论基础. 第 2 版 [M]. 人民邮电出版社. 2009. 